\title{
THE CEPHALIC NERVES: SUGGESTIONS
}

\author{
ROBERT BENNETT BEAN \\ The Anatomical Laboratory, Tulane University \\ THREE FIGURES
}

The BNA term 'cerebral' as applied to the nerves of the head is a misnomer. There are only four cerebral nerves proper, although the nerve of taste may have cerebral terminals. Hardesty, in the anatomy of Morris, returned to the use of the old term 'cranial' in preference to 'cerebral' and rightly so, because the nerves distributed in the head do pass through openings in the base of the skull, and but four of them are attached to the cerebrum.

Furthermore, the cranial nerves need a reclassification. No author or publisher of a text-book of anatomy has yet been brave enough to replace the twelve pairs of cranial nerves with the nerves as they really exist. The time is propitious for some alteration because of the gross errors and apparent complexity that exist in an attempt to conform recent discoveries with old fashioned notions.

For instance, there are not two olfactory nerves but about forty, the olfactory bulb and tract being not a nerve but an outgrowth of the brain. Likewise, the optic nerve is not a nerve but another outgrowth of the brain: There is a retina which is a modified cerebral cortex, an association tract and a decussation, but no nerve proper. The first and second pairs of cranial nerves should therefore be described with the olfactory apparatus and the optic apparatus. The tenth and eleventh cranial nerves should not be considered with the nerves of the head, because they are distributed to the neck, shoulder and trunk, and form an intermediate stage between the cervical spinal nerves and cranial (why not cephalic?) nerves. Omitting these four pairs 
of nerves there remain but eight of the usually so-named cranial nerves. However, if only one cranial nerve be lost the classic twelve is broken and all indication by numerals may as well be discarded.

I therefore propose an alteration in the terminology of the present so-called cranial nerves in the following manner:

1. Call the nerves distributed in the head the cephalic instead of the cranial or cerebral nerves. The head includes the cranium and face, with the orbital, nasal and buccal cavities as a part of the latter.

2. Omit the olfactory and optic nerves and describe them under their proper apparatuses.

3. Omit the pneumogastric and spinal accessory nerves from the cephalic group because they belong to the spinal cord type, or may be considered as transitory nerves between the spinal cord and brain, and they are not distributed to the head but to the neck, shoulders and trunk.

4. Add three nerves to the cephalic group.

a. Add the motor root of the trigeminals, which is as much a separate nerve as the facial, the two supplying motion to the mandible and face, as motor reciprocal to the trigeminal which supplies sensation to the teeth and face. The name of the motor root of the trigeminal should be the masticator nerve (n. masticatorius), as given in the BNA, because it supplies the muscles of mastication.

$b$. Add the sensory part of the facial, including the intermediate nerve of Wrisberg, the geniculate ganglion containing the cells of origin of this nerve, and the chorda tympani, with its distribution in the tongue and palate. Call this nerve the glossopalatine nerve (n. glossopalatinus), as suggested by Hardesty. The work of Streeter, Cushing, Sheldon and others demonstrates that this nerve is only a segregated portion of the glosso-pharyngeal nerve in its ontogeny and phylogeny, as well as in its central and peripheral terminations, but its roundabout course necessitates a separate name.

c. Add the nerve of the semicircular canals, separating it from the nerve of the cochlea. Retain the name acoustic or auditory 
for the latter and give the former separately the name it bears at present, vestibular nerve.

5. Finally, omit the sympathetic ganglia of the head, especially the ciliary, sphenopalatine, otic and submaxillary, from the description of the cephalic nerves, and constitute them as a ganglionated cephalic plexus, including the sympathetic part of the geniculate, petrous and jugular ganglia, the prolongation upward of the cervical sympathetic system. This will be considered separately at the end of this study.

The nerves as rearranged may be enumerated as the cephalic nerves:

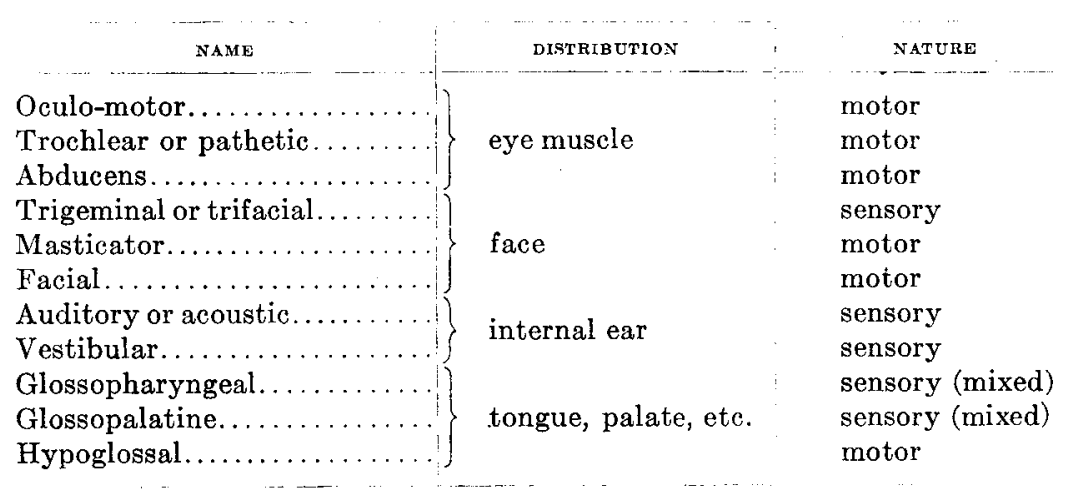

This is a more rational arrangement than that of the twelve cranial nerves, if only the grouping of the nerves of like distribution is considered and when it is kept in mind that the origin and central connections, course and distribution of each nerve is distinct, their individuality is apparent.

It may be well to give descriptions of the newly constituted nerves, the masticator nerve, the vestibular nerve and the glossopalatine nerve, although the masticator and vestibular nerves are so well known under the names of the motor root of the trigeminal, and the vestibular part of the acoustic, respectively, that only the glossopalatine nerve will be described in detail. Diagrams are given to illustrate the course and distribution of the masticator and glossopalatine nerves, and the ganglionated cephalic plexus and its connections. (figs. 1, 2 and 3). 


\section{The glossopalatine nerve}

This nerve consists of four parts which have been recognized as parts of one nerve but not so described in the text-books. The four parts are the pars intermedia or intermediate nerve of Wrisberg, the geniculate ganglion, the chorda tympani and the palatine portion of the nerve. The nerve is apparently an aberrant part of the glossopharyngeal nerve. Its cells of origin are located in the geniculate ganglion, the peripheral processes

\section{ABBREVIATIONS}

a.d.t.n., anterior deep temporal nerve a.t.n., auricular temporal nerve

b.n., buccinator nerve

c.p., carotid plexus

c.a., carotid artery

c.t., chorda tympani

$c . b$, communicating branch to middle

cervical sympathetic ganglion

$c . g .$, ciliary ganglion

c.t.n., caroticotympanic nerve (small deep petrosal)

c.c.p., carotid and cavernous plexuses

c.n., carotid nerve

e.p.n., external pterygoid nerve

e.c.a., external carotid artery

e.s.p.n., external superficial petrosal nerve

f.n., facial nerve

f.a., facial artery

f.n.f., facial nerve fibers

G.g., Gasserian ganglion

g.n., glossopharyngeal nerve

g.p. of g.g., glossopalatine portion of geniculate ganglion

gen.g., geniculate ganglion

g.s.p.n., great superficial petrosal nerve

g.d.p.n., great deep petrosal nerve

g.p.n., glossopalatine nerve

g.r., geniculotympanic ramus

i.c.t.n., inferior carotico tympanic nerve

i.c.n., internal carotid nerve

i.p.n., internal pterygoid nerve i.m.a., internal maxillary artery

i.a.n., inferior alveolar nerve

$j . n .$, jugular nerve

j.g., jugular ganglion

$l . n .$, lingual nerve

mas.n., masseter nerve

my.n., mylohyoid nerve

m.m.a., middle meningeal artery

$\operatorname{max.n.,~maxillary~nerve~}$

m.m., mandibular nerve

mas.n., masticator nerve

n. of p.c., nerve of the pterygoid canal (Vidian)

n.p., nodosal plexus

$o . g .$, otic ganglion

oc.n., oculomotor nerve

op.n., ophthalmic nerve

$p . i .$, pars intermedia

p.d.t.n., posterior deep temporal nerve

$p . n$., petrosal nerve

$p . g .$, petrous ganglion

p.p., of g.n., palatine portion of glossopalatine nerve

s.g., sphenopalatine ganglion

sub.g., submaxillary ganglion

s.c.s.g., superior cervical sympathetic ganglion

s.s.p.n., small superficial petrosal nerve

s.g., sphenopalatine ganglion

t.n., tympanopetrosal nerve

t.p., tympanic plexus

tym.n., tympanic nerve (Jacobson's)

tri.n., trigeminal nerve 
of which end in the anterior two-thirds of the tongue and the soft palate, and the central processes of which terminate about cells superior to the nucleus of termination of the glossopharyngeal nerve in the medulla.

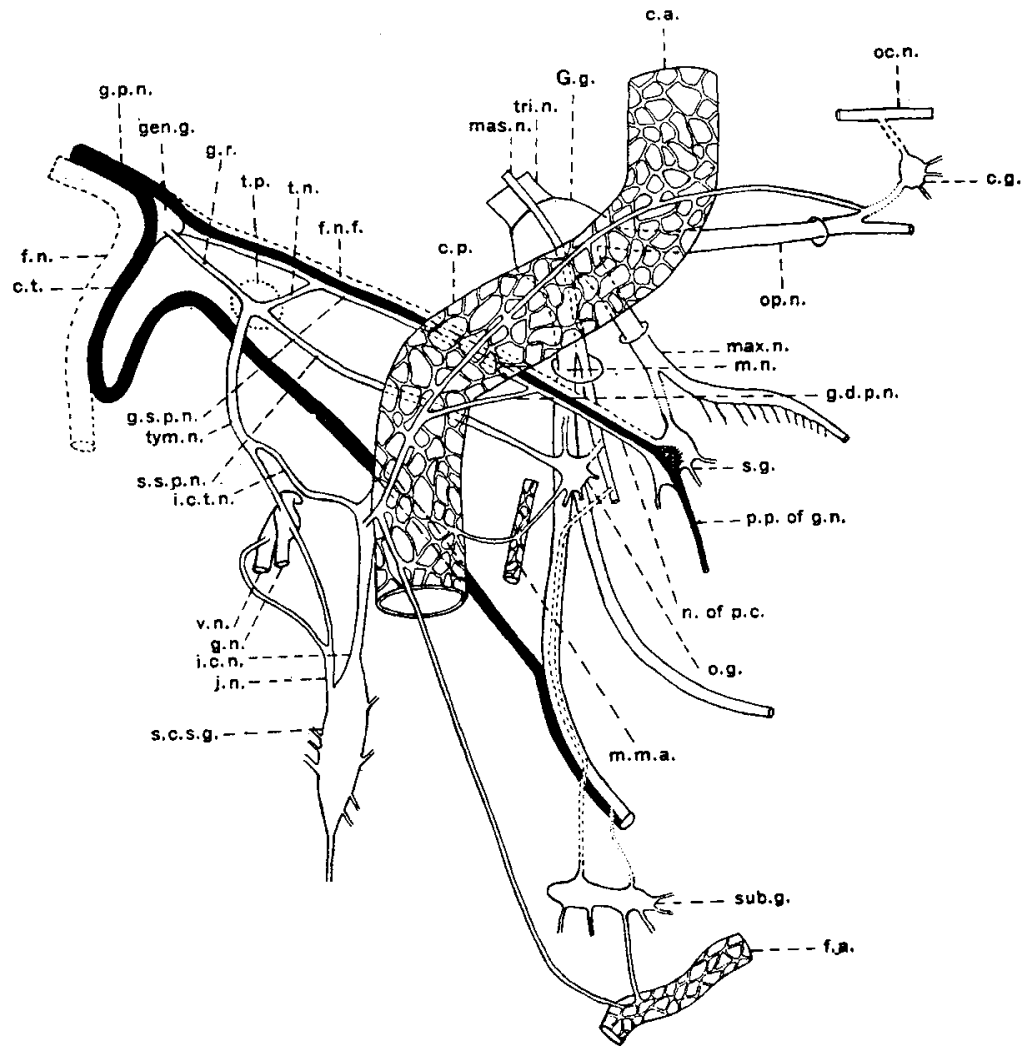

Fig. 1 Diagram showing the glossopalatine nerve and the ganglionated cephalic plexus. Each ganglion has three roots; the motor root is in broken lines, the sensory is in dotted lines and the sympathetic is in solid lines. Mesial view, left side.

The geniculate ganglion is embedded in the anterior border of the great bend of the facial nerve behind the hiatus Fallopii. It is somewhat triangular in form and at its three angles three nerves are found. Its external angle has the chorda tympani attached, its anterior angle is connected with the great superficial petrosal nerve, and its superior angle has the root of the inter- 


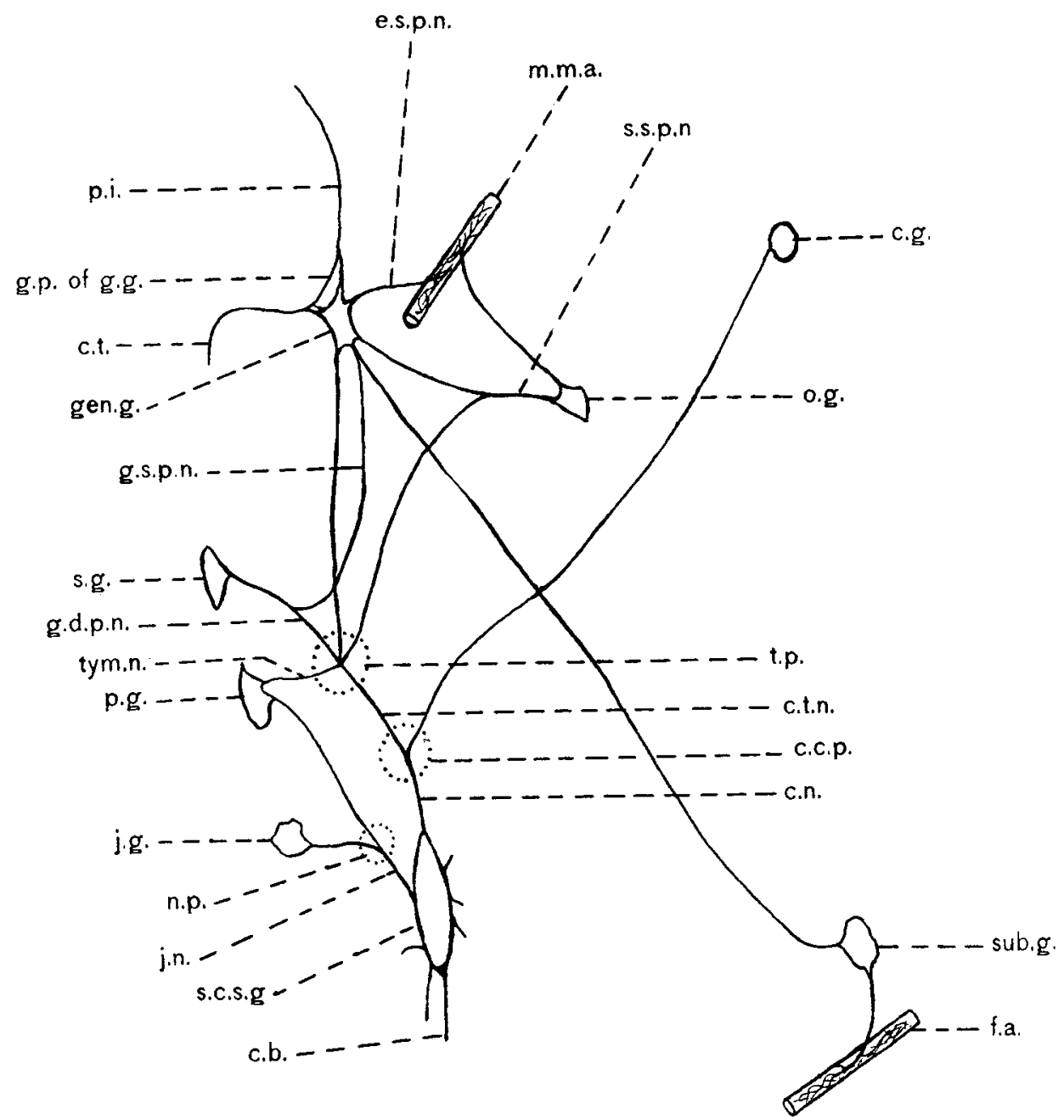

Fig. 2 Scheme to represent the continuity of the sympathetic connections of the cephalic ganglia (ganglionated cephalic plexus).

mediate nerve proper. A part of the geniculate ganglion belongs to the sympathetic system and will be described below with the ganglionated cephalic plexus.

The fibers of the intermediate nerve concerned in the glossopalatine nerve pass from the geniculate ganglion in the facial 
canal (aqueduct of Fallopius) inside the sheath of the facial nerve, which it leaves as it passes inward through the internal auditory meatus to turn slightly downward in the posterior fossa of the cranium. It enters the medulla immediately below the pons between the facial and auditory nerves, passes through the reticular formation inward and backward to terminate in the group of cells superior to the nucleus of termination of the glosso-

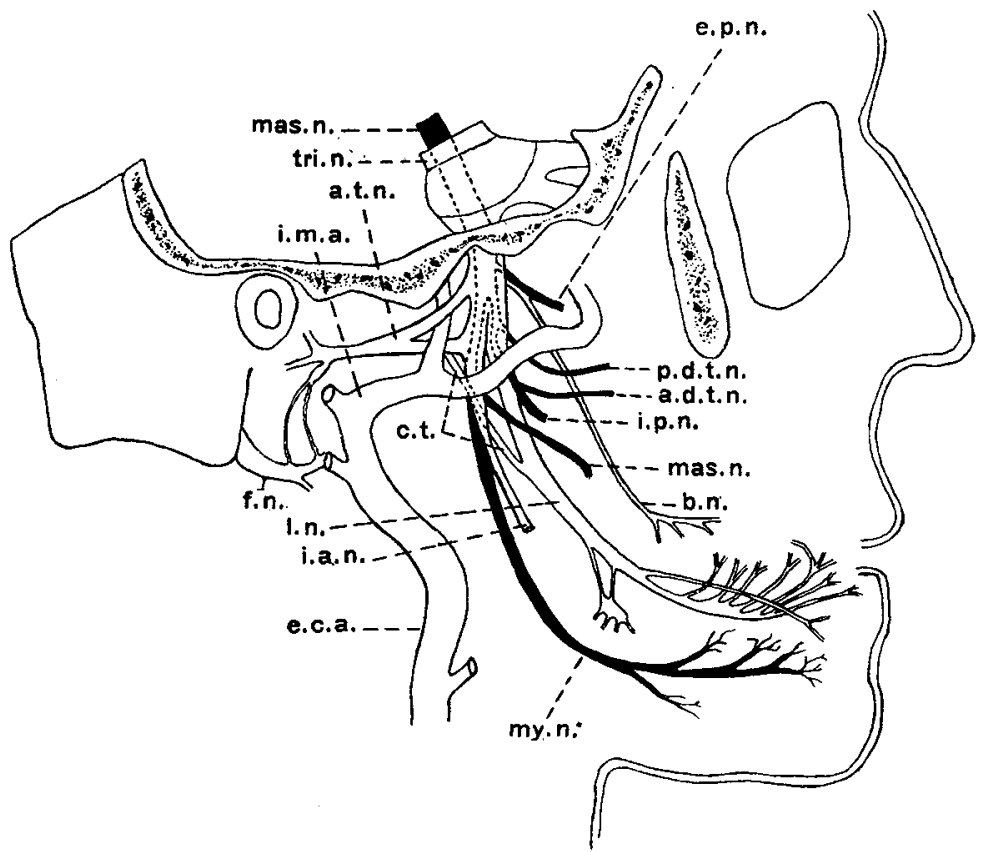

Fig. 3 Schematic representation of the masticator nerve (in black); modified from Spalteholz. Lateral view, right side.

pharyngeal nerve. In the internal auditory meatus the nerve gives two delicate filaments to the vestibular nerve. The intermediate nerve contains a few motor fibers probably acquired while it is in the sheath of the facial nerve. It also may contain secretory fibers from the medulla whose impulses reach via sympathetic neurones the glands and mucous membrane of the salivary apparatus. 
The chorda tympani is so well known it need not be described. Other sensory fibers of the glosso-palatine nerve besides those of the chorda tympani rise in the geniculate ganglion and pass through the great superficial petrosal nerve and the spheno-palatine ganglion to the soft palate where they are probably connected with the peripheral taste organs found there, as well as serving as fibers of general sensibility for the palate.

Enough has been said to indicate the most essential changes to bring the nerves of the head up to date. There is one other change, a change in the hypoglossal nerve. The ansa hypoglossi or loop of the hypoglossal is not a part of this nerve and should not be included with it but instead should be described with the cervical plexus of which it is a part. The name ansa hypoglossi should be discarded and in its place the name ansa cervicalis substituted. Likewise the descendens hypoglossi and the communicans hypglossi should be altered to the descendens cervicalis and the communicans cervicalis.

Whether the suggestions here given be followed or not there can be no doubt that such changes would clarify and simplify the cephalic nerves. The removal of the ganglia and their connections from the facial, trigeminal and glosso-pharyngeal nerves, and so forth, simplifies these nerves, and the segregation of the masticator, vestibular and glosso-palatine nerves further simplifies the nerves with which they are usually described.

The ganglionated cephalic plexus (figs. 1-2)

The ganglia of the head receive and distribute three classes of fibers, motor, sensory and sympathetic. They do not belong to any one nerve or set of nerves, although they all have sympathetic connections and may be considered as the prolongation upward of the cervical sympathetic ganglia. They correspond both structurally and developmentally with the sympathetic ganglia, except those parts of the geniculate, petrous, and jugular ganglia which represent the dorsal root ganglia, or ganglia of origin of the sensory portions of the glossopalatine, glossopharyngeal, and pneumogastric nerves respectively. Some of these ganglia of the head are vagrant ganglia which separated from the 
embryonal semilunar or Gasserian ganglion at an early period of development--just as the sympathetic ganglia of the neck and trunk separated from the embryonal spinal ganglia.

These ganglia of the head with their connections may be conveniently grouped together as the ganglionated cephalic plexus, and may be said to consist of the ciliary, the sphenopalatine or Meckel's ganglion, the otic, the submaxillary, and a part of the geniculate, of the petrous and of the jugular ganglion.

Each ganglion may be said to possess a motor root, a sensory root, and a sympahetic root, and the ganglia act as relays and points of dispersal for these three sets of fibers. Motor impulses go to the eyeball, the palate, the middle ear and the pharynx; sensory impulses come from the same regions and from the lining of the mouth; and secretory fibers are distributed to the lachrymal glands, to the mucous membrane of the nose and mouth, pharynx and palate, to the parotid glands, and to the submaxillary and sublingual glands.

The superior cervical sympathetic ganglion gives off two large branches which pass upward to communicate with the ganglionated cephalic plexus. One of these, the carotid nerve, is the direct continuation upward of the gangliated cord, and through its branches, the caroticotympanic and the deep petrosal nerves, as well as through the plexuses derived from it, this nerve communicates with practically all the ganglia of the head. Another nerve from the superior cervical sympathetic ganglion, the jugular nerve, passes directly to the ganglia of the pneumogastric and glossopharyngeal nerves, through the nodosal plexus to the jugular ganglion, and directly to the petrous ganglion.

It may be well to describe each ganglion with its roots of origin, and its branches of distribution, in order to obtain a clear insight into the composition of the ganglionated cephalic plexus. Whereas the three roots will be described in the terminology of gross anatomy, it is realized that in their microscopic construction the roots of the sympathetic portions of the jugular, petrous and geniculate ganglia especially, may be of varying significance. Very few fibers of the sensory root of a sympathetic ganglion actually terminate within the ganglion, about its cells, 
and probably none except the few which terminate in its capsule, and in which arise general sensations from the ganglion to the central system. A motor root of a sympathetic ganglion may consist of two varieties of fibers; those which arise in the motor nuclei (of origin) in the central system and terminate about cells within the sympathetic ganglion (splanchnic efferent fibers) and, second, those which have the same origin and pass through the ganglion uninterrupted to their termination upon striated muscle (somatic efferent fibers), or pass through and go as splanchnic efferent fibers to other sympathetic ganglia. A sympathetic root of a ganglion may consist of two or even three varieties of fibers: (1) those which arise from cells in other sympathetic ganglia and terminate about cells in the ganglion in question, which cells in turn contribute fibers to its branches of distribution; (2) fibers arising in other sympathetic ganglia which pass through the ganglion uninterrupted, and out with its branches of distribution; and (3), in case of the mixed ganglia of cephalic and spinal nerves especially, fibers arising in sympathetic ganglia which enter the ganglion in question and terminate about cell bodies of the dorsal root ganglion type, that is, cell bodies giving origin to ordinary cerebro-spinal sensory fibers, which are thus enabled to carry into the central system sensory impulses arising in the sympathetic distribution, as well as sensations arising in the cerebro-spinal peripheral terminations. The present knowledge of the minute construction of the ganglia of the ganglionated cephalic plexus and the origin of the fibers related to them in the plexus renders it impossible to use a terminology in describing them based upon their construction.

\section{The jugular ganglion}

This ganglion lies on the trunk of the vagus nerve in the jugular foramen. It receives its motor and sensory roots from the vagus nerve and its sympathetic root from the superior cervical sympathetic ganglion through the nodosal plexus immediately inferior to it.

Its chief nerve of distribution is the auricular branch of the vagus, or nerve of Arnold, which leaves the jugular ganglion in 
the jugular foramen. It receives a branch from the petrous ganglion, enters the mastoid canaliculus in which it receives a communication from the facial nerve or merely lies in contact with it as far as the stylomastoid foramen, where it leaves the temporal bone. It may pass through the tympanomastoid fissure, after which it divides behind the pinna into two branches, one of which joins the posterior auricular branch of the facial, and the other ramifies in the posterior inferior part of the cartilaginous portion of the ear, and to the skin of the dorsal part of the pinna. It also supplies twigs to the bony part of the external auditory meatus, and to the lower part of the outer surface of the tympanic membrane.

\section{The petrous ganglion}

This ganglion lies around the glossopharyngeal nerve in the lower part of the jugular foramen. The motor and sensory roots are derived from the vagus and probably also from the facial and glossopharyngeal nerves. The sympathetic root is a fine filament derived from the superior cervical sympathetic ganglion.

The branches of distribution of the petrous ganglion are mainly through the tympanic branch, or nerve of Jacobson, to the tympanic plexus where communication is established with the facial nerve, and with two branches of the carotid nerve, the superior and inferior caroticotympanic nerves which enter the tympanic cavity through channels of the same name. From this plexus arises the small superficial petrosal nerve which goes to the otic ganglion. Other branches are distributed to the middle ear, and many filaments pass to the carotid plexus, while still others go to the pharyngeal plexus. Fibers also pass by way of the glossopharyngeal nerve to the tonsils.

The tympanic plexus serves as a common point of distribution of fibers from the carotid plexus, the cavernous plexus, and the superior cervical sympathetic ganglion, by way of the caroticotympanic nerves, and the jugular and tympanic nerves. The tympanic plexus communicates with the petrous, jugular, geniculate, otic and sphenopalatine ganglia, and the only cephalic ganglia of the sympathetic that it does not communicate with 
are the ciliary and submaxillary ganglia, which are supplied from the carotid plexus direct, or from plexuses derived from the carotid. The tympanic plexus is formed by nerves derived indirectly from the superior cervical sympathetic ganglion, the inferior caroticotympanic from the carotid plexus, the superior caroticotympanic from the cavernous plexus, and the tympanic nerve derived from the petrous and jugular ganglia which it has entered as the jugular nerve. The tympanic plexus communicates with the geniculate ganglion through a small branch which may be called the geniculotympanic ramus; it communicates with the sphenopalatine ganglion through the great superficial petrosal nerve by way of a branch that may be called the tympanopetrosal ramus; and it communicates with the otic ganglion by way of the small superficial petrosal nerve. It will be seen that the tympanic plexus with the carotid and derivitive plexuses and the cephalic ganglia enumerated above represent broken up cephalic portions of the gangliated sympathetic cord whose ganglia have fused more or less in the neck and have become scattered in the head. The tympanic plexus is the communicating plexus of the ganglia, and represents not only the rami communicantes, but the sympathetic trunks connecting the sympathetic ganglia. The ganglia of the glossopalatine, glossopharyngeal and pneumogastric nerves, that is, the geniculate, petrous and jugular ganglia, are different from the other cephalic sympathetic ganglia, in that the central and sympathetic portions have not become separated by the wandering off of the sympathetic portions. The ciliary, sphenopalatine, otic and submaxillary ganglia wandered off from the Gasserian ganglion at an early embryonic period, in a manner similar to the wandering of the sympathetic ganglia of the gangliated cord from the spinal root ganglia, and the four ganglia are true sympathetic ganglia. The sympathetic portions of the ganglia of the glossopalatine, glossopharyngeal and pneumogastric nerves remain fused with the spinal portions of the same ganglia, but the tympanic plexus forms a common ground of communication, and affords a ready means of understanding the relationship between all the cephalic sympathetic ganglia. 
The geniculate ganglion

This ganglion lies embedded in the anterior border of the geniculum of the facial nerve dorsal to the hiatus Fallopii. Its motor root is of fibers from the facial nerve joining it at the great bend of the latter. The sensory root is derived from the dorsal root portion or the ganglion of the glossopalatine nerve, either in the ganglion or adjacent to it. The sympathetic root is the external superficial petrosal which passes through the hiatus Fallopii and connects the ganglion with the sympathetic plexus on the middle meningeal artery or through the great superficial petrosal nerve from the sphenopalatine ganglion. The branches of distribution of this ganglion pass through other ganglia, or other nerves. The great superficial petrosal nerve passes directly from the geniculate ganglion to the sphenopalatine ganglion. A communicating branch passes from the geniculate ganglion to the small superficial petrosal nerve which afterwards enters the otic ganglion.

\section{The sphenopalatine or Meckel's ganglion}

This ganglion and the three following may be described as in the anatomy of Morris, remembering to dissociate them from the trigeminal nerve. The important facts concerning these ganglia are to determine their motor, sensory and sympathetic roots of origin, and to establish their fibers of distribution. The three roots of origin, motor, sensory and sympathetic, of the sphenopalatine ganglion are from the facial, trigeminal, and great deep petrosal nerves respectively. The fibers are distributed to the mucous membranes lining the ethmoidal and sphenoidal sinuses, and that of the nasal, buccal and pharyngeal cavities. Fibers of taste belonging to the glossopalatine nerve, arising from the geniculate ganglion, pass through the sphenopalatine ganglion from the great superficial petrosal nerve, and are distributed to the soft palate. Some of the small palatal muscles may be supplied by motor branches passing through this ganglion without interruption. 
The position of the sphenopalatine ganglion is determined by the size and shape of the sphenoid sinus. In many recent dissections I have found it medial, lateral or inferior to this sinus and in five subjects the Vidian nerve passed through the sphenoid sinus in a tube of bone covered with mucous membrane.

\section{The otic ganglion}

The motor root of the otic ganglion is derived from the motor nucleus of the facial and some fibers probably also from the masticator nerve. Its sensory root passes through it from the glossopharyngeal nerve, and its sympathetic root from the middle meningeal plexus and from the tympanic plexus through the continuation of the tympanic nerve in the small superficial petrosal nerve. The sympathetic fibers from the otic ganglion are distributed to the parotid gland, the glands and vessels of the tongue, and uninterrupted motor cranial fibers to the tensor tympani and tensor veli palatini muscles.

\section{The submaxillary ganglion}

The motor root of this ganglion is derived from the motor nucleus of the glossopalatine, the sensory root from the lingual (Gasserian ganglion) and the sympathetic root from the plexus on the facial artery, or from the sympathetic portion of the geniculate ganglion through the chorda tympani. The fibers arising from this ganglion are distributed to the submaxillary and sublingual glands and to the floor of the mouth.

\section{The ciliary ganglion}

The motor root of this ganglion is derived from the oculomotor nerve, fibers which terminate in it, the sensory from the trigeminal, and the sympathetic root from the cavernous plexus. The fibers from this ganglion are distributed to the ciliary body, the iris, the cornea, and probably to the lachrymal gland. 
The parotid ganglion

A small plexiform mass containing ganglion cells, and located beneath the parotid gland on the auriculo-temporal nerve, connecting with the vagus, facial and trigeminal nerves, has been dissected repeatedly by students in the laboratory at Tulane University under my direction. At present investigations are under way to determine if this is the ganglion of the parotid gland. I realize that the term sympathetic needs restriction in its use, therefore, I propose that it be reserved for use in gross description, the sympathetic nervous system as a whole, in the same way that the terms central and peripheral nervous systems are used. In any presentation of the functional differences of neurones the terms somatic motor, somatic sensory, visceral motor and visceral sensory should be used insofar as present knowledge of the subject will permit.

From the standpoint of gross anatomy there can be no question as to the propriety of assembling the ganglia of the head and their connections as the ganglionated cephalic plexus, and from the standpoint of their sympathetic connections they are one, although it is desirable to determine exactly what fibers are motor, sensory or secretory, where they have their origin and where they are distributed. 